\title{
A Study on Drinking Motives Among College Students in Chennai City-A Cross Sectional Exploratory Survey
}

\author{
Gururajan Mohan \\ M.PHIL (Sociology) \\ Jawaharlal Institute of Postgraduate Medical Education and Research, Pondicherry, India
}

\section{INTRODUCTION}

Alcohol is a substance which is widely used throughout the world and has been present for centuries. Its unique effects on the human body and mind have drawn attention and lead to extensive research being done on its effects. It is an illness characterized by significant physiological, psychological and or social dysfunctions associated with persistent and excessive use of alcohol. It is a chronic and progressive disease featured by loss of control over the use of alcohol with subsequent social, legal, psychological \& physical consequences. Alcohol consumption has risen greatly during the last 4 decades and has been accompanied by increase in the social, psychological and physical problems. The term 'alcoholism' is now used in a broad sense to describe a pattern of drinking which is harmful to the individual or to his or her family. There are many factors which lead a cause for alcohol dependence such as genetic factors, cultural factors, availability, economical barriers, emotional pleasure, and physical dependence.

Alcohol dependence is a major health problem in India. An estimated $32-42 \%$ of adult Indian population reports high usage of alcohol in their lifetime. 5-7\% is abuser of alcohol and 10- 20 million persons have been estimated to be in need of treatment for alcohol dependence. The reports also indicated that there is a steady rise in per capita alcohol consumption every year.

In India, drinking seems to be a male habit and considered to be a major public health issue. Drinking among college students continues to be a considerable problem; College students often face novel social situations in a context of heightened accessibility to alcohol and frequent promotion of excessive drinking (Ham \& Hope, 2003).Students are at the stages of their life to perform and to achieve; such drinking motives may spoil their career and may lead to addiction. Also excessive drinking among college students is associated with damaged property, poor class attendance, hangovers, trouble with authorities and injuries (Wechsler, Davenport, Dowdall, Moeykens, \& Castillo, 1994), and violence (White, 1997).

Excessive drinking among college students is associated with damaged property, poor class attendance, hangovers, trouble with authorities, and injuries (Wechsler et al., 2000; Wechsler, Davenport, Dowdall, Moeykens, \&Castillo, 1994). Alcohol consumption is prevalent among college students, and there are many reasons for its use and abuse (Luhtanen \& Crocker, 2005). However, for successful efforts to limit premature and excessive drinking among adolescents it is necessary to understand the antecedents and etiology of drinking behavior. In this regard, the motivation for engaging in drinking is one important aspect. Theorists regard drinking motives as the final common pathway to its use, i.e. the gateway through which more distal influences are mediated (Cooper, 1994; Cox \& Klinger, 1988). Surprisingly, the prevalence of alcohol consumption in college populations has remained consistent, though the rate of binge drinking has increased (Wechsler, 2000). Researchers have found several factors influencing alcohol use such as social pressures, social anxiety, spirituality, and many other aspects of the human selfconcept (Neighbors,Larimer, Geisner, \& Knee, 2004, Bruch, Gorsky, Collins, \& Berger, 1989, Luhtanen et al, 2005). 
Historically, consumption of alcohol was totally banned in Tamil Nadu state between 1937 to 1991 and the ban was lifted only 3 times during those period. The whole production and sale of alcohol is controlled by the Government Company called TASMAC (Tamil Nadu State Marketing Corporation) which has nearly 7000 sale outlets and about 30,000 employees. There has been a steady profit over the years. Reports suggest that in 2005-06, the annual revenue was about 7,335 crores of Indian rupees (73.35 billion rupees, equivalent to $\$ 1336$ million). The most recent data (2011-12) showed annual revenue of an astonishing 18,018 crores of Indian rupees of 180.18 billion rupees, roughly equivalent to $\$ 3.3$ billion. (Times of India, 2012) Particularly in the metropolitan city of Chennai, alcohol use disproportionately harms chennai males, because the affordability and availability of the alcohol is more.

In the state of Tamilnadu manufacturing of the alcohol such as brandy, arrack and toddy are some of the important businesses. Also the cost of the alcohol is very low in the neigh boring state of Pondicherry when compared to the other states in India. Adolescents and young adults in the country are highly vulnerable to the onset and continuation of the habit. Especially, male college students are more prone to the alcohol abuse because due to Sociocultural factors, such as urbanization and westernization and environmental factors.

Research has also demonstrated links between college drinking and unwanted sexual advances, unplanned and unprotected sex, sexual aggression, and sexual assault (Frintner \& Rubinson, 1993; Koss \& Gaines, 1993; Larimer, Lydum, Anderson, \& Turner, 1999; Wechsler et al., 2000, Wechsler et al., 1994). To our knowledge this topic was not given much importance in Indian context due to the fact that this is restricted to western individualistic culture. However, globalization and technological advancement have made people from developing nations comes in contact with the individualistic nations and as a result to adapt the culture of western nations. In particular, any city in a developing nation with multiple cultural mixes, there are higher chance of adapting such western cultural practices as easily. The main focus of this study is to identify the drinking motives among college students in a multicultural and the capital city of Tamilnadu, which was formerly called as Madras, using motivational model of alcohol use (Cox and Klinger, 1988)
Motivational model of alcohol use an overview: Motivational models of alcohol use propose that individuals are motivated to drink alcohol in order to reach a particular goal state (e.g. tension reduction; Cox \& Klinger, 1988). Drinking motives are the proximal reasons underlying alcohol use and are believed to serve as the final "common pathway" through which more distal factors (e.g. depression, anxiety) exert their influence (Cooper, 1994). Cooper proposed four classes of drinking motives: enhancement (e.g. "Because you like the feeling"), social (e.g. "Because it makes social gatherings more fun"), coping (e.g. "To forget your worries"), and conformity (e.g. "To fit in with a group you like"). The negative reinforcement motives of coping and conformity are associated with utilizing alcohol to avoid negative outcomes, such as negative affect or social censure. The positive reinforcement motives of enhancement and social entail using alcohol to increase positive affect or as part of social functions to increase enjoyment. Research suggests that coping and conformity motives have been associated with alcohol-related problems above and beyond alcohol consumption levels, while enhancement motives were only related to alcohol related problems via increased heavy drinking and social motives were unrelated to alcohol related problems (Carpenter \& Hasin, 1999; Cooper, 1994).

The concept of drinking motives is based on the assumption that people drink in order to attain certain valued outcomes (Cooper, 1994; Cox \& Klinger, 1988). It also assumes that drinking behavior is motivated by different needs or serves different functions, and that specific drinking motives are associated with a unique pattern of precursors and consequences. Heavy drinking, for example, is particularly likely among people who experience stress and drink for coping motives, as well as those whose friends drink heavily and who drink for social motives themselves (Abbey, Smith, \& Scott, 1993). In other words, drinking motives or reasons represent a subjectively derived decisional framework foralcohol use based on personal experience, situation and expectancies (Carpenter \& Hasin, 1998b; Cox \& Klinger, 1988).

The motivational model assumes that a person makes a decision about whether or not he or she will consume alcohol. The decision to drink is a combination of emotional and rational processes in that the decision is made on the basis of the affective 
change that the person expects to achieve by drinking compared with not drinking. The affective change can either be related to the direct chemical effects of alcohol, e.g. tension reduction or mood enhancement, or the indirect effects, such as peer acceptance. In fact, a person does not have to be aware of either having made a decision to drink or the factors affecting this decision. In most cases, decisions about drinking are even unconscious and automatized. According to the model, the decision to drink is embedded in historical and current factors, expected effects, and drinking motives.

Historical factors relate to biochemical reactivity to alcohol (e.g. the genetic disposition to react positively or negatively to alcohol), personality characteristics (e.g. non- conformity, impulsivity, extraversion, sensation seeking, or self-derogation), socio-cultural and environmental factors (e.g. culture-specific drinking styles), and past reinforcement from drinking. Current factors are associated with quality of life in terms of the quantity and quality of prevailing positive and negative incentives for drinking and situational factors (e.g. if alcohol is available or being exposed to people who drink).

According to motivational model of alcohol use (Cox and Klinger, 1988) people use to drink to obtain positive outcomes (positive reinforcement) or to avoid negative consequences (negative reinforcement) and they may be motivated by internal rewards such as enhancement of a desired internal emotional state or by external rewards such as social approval or acceptance.

Four different drinking motive categories were developed when crossing these two dimensions. (i) internally generated, positive reinforcement motives (drinking to enhance positive mood); (b) externally generated, positive reinforcement motives (drinking to obtain social rewards); (c) internally generated, negative reinforcement motives (drinking to reduce negative emotions), and (d) externally generated, negative reinforcement motives (drinking to avoid social rejection). Using this model Cooper (1994) developed the Drinking Motives Questionnaire (DMQ) that reliably measures the four drinking motives categories and has been used in many cultures.

Drinking motives questionnaire is the most often applied multidimensional instrument to assess drinking motives. However, urbanization and globalization has increased use of alcohol even in developing countries with high cultural regulations. India is not an exception for this issue, for instance availability of low cost and tax reduced alcohol can be a factor which triggers drinking motives in Chennai, one of the metro-politan city in India. To our knowledge no study exists that has used the DMQ to assess drinking motives in the city of Chennai. Even though alcohol consumption and drinking patterns among adolescents vary widely across cultures, it is highly interesting to note the drinking motives of nonwestern population which try to imitate the western world.

All though alcohol consumption and drinking patterns among college students differs across cultures, we tried to use DMQ to find out the applicability in Indian context.

\section{DRINKING MOTIVES:}

Drinking motives are defined as the final decision whether to drink or not to drink and therefore the most proximal factor for engaging in drinking(Cox and Klinger, 1988). On the other way, drinking motives are the final pathway to alcohol use, i.e the gateway through which more distal influences, such as personality characteristics are mediated (Cooper, Frone, Russell, and Mudar 1995).

Although drinking motives are associated with drinking in different situational contexts, they explain a substantial amount of variance in alcohol use in addition to situational factors such as drinking circumstance, location, day of the week, group size, type of relationship, local norms and residence (Kairouz, Gliksman, Demers, \& Adlaf, 2002).The concept of drinking motives is based on the assumption that people drink in order to attain certain valued outcomes (Cooper, 1994; Cox \& Klinger,1988). It also assumes that drinking behavior is motivated by different needs or serves different functions, and that specific drinking motives are associated with a unique pattern of precursors and consequences. Heavy drinking, for example, is particularly likely among people who experience stress and drink for coping motives, as well as those whose friends drink heavily and who drink for social motives themselves (Abbey, Smith, \& Scott, 1993). In other words, drinking motives or reasons represent a subjectively derived decisional framework for alcohol use based on personal experience, situation and 
expectancies (Carpenter \& Hasin, 1998b; Cox \& Klinger, 1988).

In order to deter excessive drinking among college students and prevent unwanted consequences, it is important to understand what motivates college students to drink. Drinking motives can be considered proximal antecedents of drinking behavior and have been shown to predict alcohol consumption and alcohol-related consequences (Cooper, 1994; Cooper, Russell, Skinner, \& Windle, 1992; Cooper, Frone, Russell, \& Mudar, 1995; Stewart, Loughlin, \& Rhyno, 2001). Research concerning drinking motives has identified a four-factor model including affect enhancement, coping, social rewards, and conformity (Cooper, 1994; MacLean \& Lecci, 2000; Stewart \& Devine, 2000; Stewart et al., 2001).

Affect enhancement motives refer to drinking in order to experience positive emotions, to feel good, or to experience excitement. Coping motives refer to drinking that is motivated by a desire to escape internal negative experiences such as anxiety, depression, or uncertainty. Social rewards motives are roughly analogous to drinking as a social lubricant to help one be sociable and enjoy social gatherings. Conformity motives consist of drinking as a result of implicit or explicit social pressure. These motives have been described as two dimensions based on whether the source of the expected outcome is internal (enhancement and coping) or external (social rewards and conformity) and whether outcome expectancies are positively reinforcing (enhancement and social rewards) or negatively reinforcing (coping and conformity). We suggest that they can also be viewed as a means of regulating affect (enhancement and coping) and social functioning (social rewards and conformity).

Thinking of alcohol as a means of regulating positive and negative emotions has shown much promise and has been mapped directly to enhancement and coping motives respectively (e.g., Cooper et al., 1995; Armeli, Carney, Tennen, Affleck, \& O'Neil, 2000). Mohr and colleagues (2001) have demonstrated links between positive affect and social drinking and between negative affect and solitary drinking. However thinking of alcohol as a means of regulating social functioning has not been directly evaluated.

We suggest that drinking to improve social functioning or to avoid being rejected by peers is, in essence, social regulation. There are various ways and instruments to measure drinking motives. A first group of studies adopted a qualitative approach whereby they simply asked young people why they drink.

The resulting answers were subsequently classified into categories of commonly indicated reasons, like drinking to get drunk or to be part of a peer group. Only five studies could be identified that fall into this category (Alvarez \& del Rio, 1994; Boys, Marsden, \& Strang, 2001; Kloep, Hendry, Ingebrigtsen, Glendinning, \& Espnes, 2001; Lo \& Globetti,2000; Palmqvist, Martikainen, \& vonWright, 2003).A second group of studies collected several items on drinking reasons from previous research without explicitly classifying them into broader motive categories, factors or dimensions. In this category, nine studies could be identified (De Micheli \& Formigoni, 2002; Feldman, Harvey, Holowaty, \& Shortt, 1999; Jerez \& Coviello, 1998; Klein, 1992; Montgomery, Benedicto, \& Haemmerlie, 1993; Neff, 1997; Plant, Bagnall, \& Foster, 1990; Rutledge \& Sher, 2001; Webb, Getz, Baer, \& McKelvey, 1999).

A third group of studies developed their own multidimensional questionnaire to measure drinking motives (e.g. Bradley, Carman, \& Petree,1991; Carpenter \& Hasin, 1998b; Cronin, 1997; Haden \& Edmundson, 1991;Kairouz et al., 2002; Karwacki \& Bradley, 1996; Labouvie \& Bates, 2002;Stewart \& Power, 2002; Weinberger \& Bartholomew, 1996; Windle \& Windle, 1996). In these developments, scales consisting of 10 to 40 items grouped into between 2 and 10 categories, dimensions, or factors were analyzed. Finally, a fourth group of studies used previously developed, evaluated,and established questionnaires, such as the Drinking Motive Questionnaire(DMQ, Cooper, Russell, Skinner, \& Windle, 1992), the Drinking Motive Questionnaire Revised (DMQ, Cooper, 1994), the Reasons for Drinking Questionnaire (RFDQ, Farber, Khavari, \& Douglass, 1980), the Reasons for Drinking Scale (RDS, Carpenter \& Hasin, 1998b), and the Social Context of Drinking Scales (SCDS, Thombs, Beck, \& Pleace, 1993). Among these multidimensional instruments, the Drinking Motive Questionnaire in its original or revised form is the most commonly used.

\section{OBJECTIVES OF RESEARCH:}

Drinking motives are defined as the final decision whether to drink or not to drink and therefore the most 
proximal factor for engaging in drinking. On the other way, drinking motives are the final pathway to alcohol use, i.e the gateway through which more distal influences, such as personality characteristics are mediated. The objectives of education is to find and to provide the intervention for the students who in taking alcohol.

The following major objectives are

$>$ To identify the drinking motives among the male college students in the Metro-politan City

$>$ To identify academic performance among male college students in Metro-politan city

$>$ To compare the level of drinking motive with demographic variable

\section{HYPOTHESIS:}

Drinking motive will be more in low academic students

\section{NEED FOR THE STUDY:}

Nowadays, Drinking has becoming more problematic behavior among college students in the India. This is mainly due to the influence of the factors like sociocultural factors, such as urbanization and westernization and environmental factors, such as availability and affordability. Especially, in Chennai these kind of behavior among students are seems to be increased due to modernization. Many researchers are thinking about the important motives for drinking among students population. Tomorrow's world is in the hand of today's young generations. College age is an important period in a student's life. This stage roughly includes the age between 18-24 years, where they are young, energetic, and enthusiastic and directed toward life goals.

Research reveals the there is a close relation between the students' self-esteem and drinking motives. It is accepted that there is an urgent need to understand and manage students so on. Hence, it is imperative to study the effect of self esteem on drinking motives among college students.

\section{STATEMENT OF THE PROBLEM:}

The present problem is stated as "drinking motives among college students". This study is designed to investigate whether there is any difference in the drinking motives and self esteem among the students based on age, order of birth, course, residence, pocket money, and onset of drinking. Due to shortage of time the research may not be able to include other psychosocial variables ii this study. This being a psychological study with limited population In particular region may not reflect the other regions /nations.

\section{RESEARCH METHODOLOGY}

This study adopts the normative survey which is descriptive and associational in nature. This study measures the drinking motives of the students. Also, it explores the various drinking motives of the students. Further, this study examines the truth of statistical hypothesis by systematically finding the difference due to their residence, difference due to their pocket money, difference due to their onset on drinking, and the with reference to drinking motives and self esteem. Above all, this study assesses the impact of self esteem on the drinking motives of the students.

\section{SAMPLE DESCRIPTION:}

In this present study, data were collected from 74 students studying in three colleges in Chennai. Students from each college were chosen on purposive sampling from three colleges were included in this study. Among them 47 were 20 and below 20 and 27 were above 20 years. 25 students belong to engineering, 23 belong to nursing and 26 belong to arts and Sciences College.

\section{TOOLS USED:}

In order to test the spelt out hypotheses, data were collected through survey method in the present study. The following standardized questionnaires were used in the survey along with the personal data sheet which explores the demographic and social variables of the subject.

$>$ The Drinking Motive Questionnaire Revised [DMQ-R,]

\section{The Drinking Motive Questionnaire Revised [DMQ-R,]:}

The Drinking Motives Questionnaire-Revised (DMQ-R; Cooper, 1994) was used to assess principal reasons for alcohol use. This 20-item, four-factor measure assesses enhancement (e.g., "because it gives you a pleasant feeling"), social (e.g., "to celebrate a special occasion with friends"), conformity (e.g., "to fit in with a group you like"), and coping (e.g., "to forget about your problems") motives for drinking. Participants indicate on a 5-point scale ranging from 1 (almost never/never) to 5 (almost always/always) the frequency with which they drink for each reason. Subscale scores are then computed by averaging the ratings across each of the five items related to each 
factor. The DMQ-R evidences good internal consistency (e.g., _s _ .91, 91, .79, .81) for enhancement, social, conformity, and coping, respectively in the present sample), and validity, and has been successfully utilized in prior research with adolescents (Comeau, Stewart, \& Loba, 2001; Dixon, Leen-Feldner, Ham, Feldner, \& Lewis, 2009; Kuntsche et al., 2008).

\section{METHOD OF DATA COLLECTION}

The researcher personally visited all the colleges and established rapport with the subjects. The selected subjects were seated comfortably and were given the booklet that contains "coopers et al drinking motives questionnaire revised (DMQ-R) and Rosenberg self esteem questionnaire". The subjects were given the instructions as per the manual, which would enable subject to perform the test at ease ad truth. Though it look much time and expense the investigator collected all the data under his personal supervision. Out of 90 data collected some of them were found to be incomplete and hence, only 74 data were taken for final analysis.

\section{ANALYSIS AND INTERPRETATION}

Table Distribution of sub samples based on the course $(N=74)$ :

Table No.4.1.1

\begin{tabular}{|c|c|c|c|}
\hline \hline \multicolumn{2}{|c|}{ Course } & Frequency & Percent \\
\hline \multirow{4}{*}{} & BA & 16 & 21.6 \\
\cline { 2 - 4 } & BSC & 23 & 31.1 \\
\cline { 2 - 4 } & BE & 25 & 33.8 \\
\cline { 2 - 4 } & BCOM & 10 & 13.5 \\
\cline { 2 - 4 } & Total & 74 & 100.0 \\
\hline
\end{tabular}

Entire sample of 74 was classified into sub samples based on personnel information obtained from students. The entire study sample belongs to a homogenous that is only male students.

Figure Bar diagram showing the distribution of sub samples based on course $(\mathrm{N}=74)$

Graph No.4.1.1

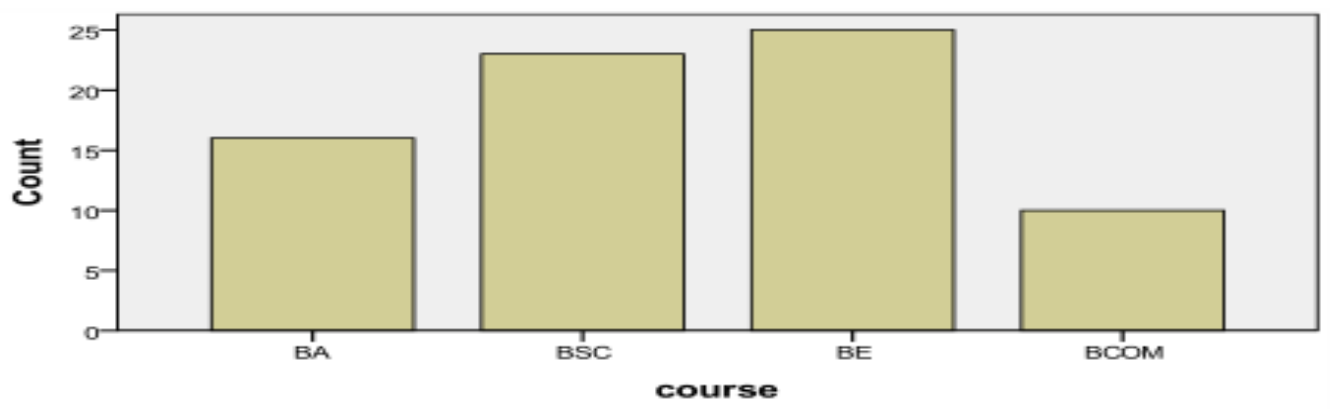


International Journal of Trend in Scientific Research and Development (IJTSRD) ISSN: 2456-6470

Table Distribution of sample and percentage on age $(\mathrm{N}=74)$

Table No.4.1.2

\begin{tabular}{||c|c|c|c||}
\hline \hline \multicolumn{2}{|c|}{ Age } & Frequency & Percent \\
\hline \multirow{3}{*}{} & $18-20$ & 47 & 63.5 \\
\cline { 2 - 4 } & $21-22$ & 20 & 27.0 \\
\cline { 2 - 4 } & $23-24$ & 5 & 6.8 \\
\cline { 2 - 4 } & $25-26$ & 2 & 2.7 \\
\cline { 2 - 4 } & Total & $\mathbf{7 4}$ & $\mathbf{1 0 0 . 0}$ \\
\hline
\end{tabular}

The above table represents the subject. The sample was divided into four groups according to age. The table reveals that 47 subjects were coming under $18-20$ of age (63.5\%), 20 subjects were coming under $21-22$ of age $(27 \%), 5$ subjects were coming under $23-24$ of age $(6.8 \%), 2$ subjects were coming under $25-26$ of age (2.7\%).

Figure Bar diagram showing the distribution of sub samples based on the age group of students $(\mathrm{N}=74)$

Graph No.4.1.2

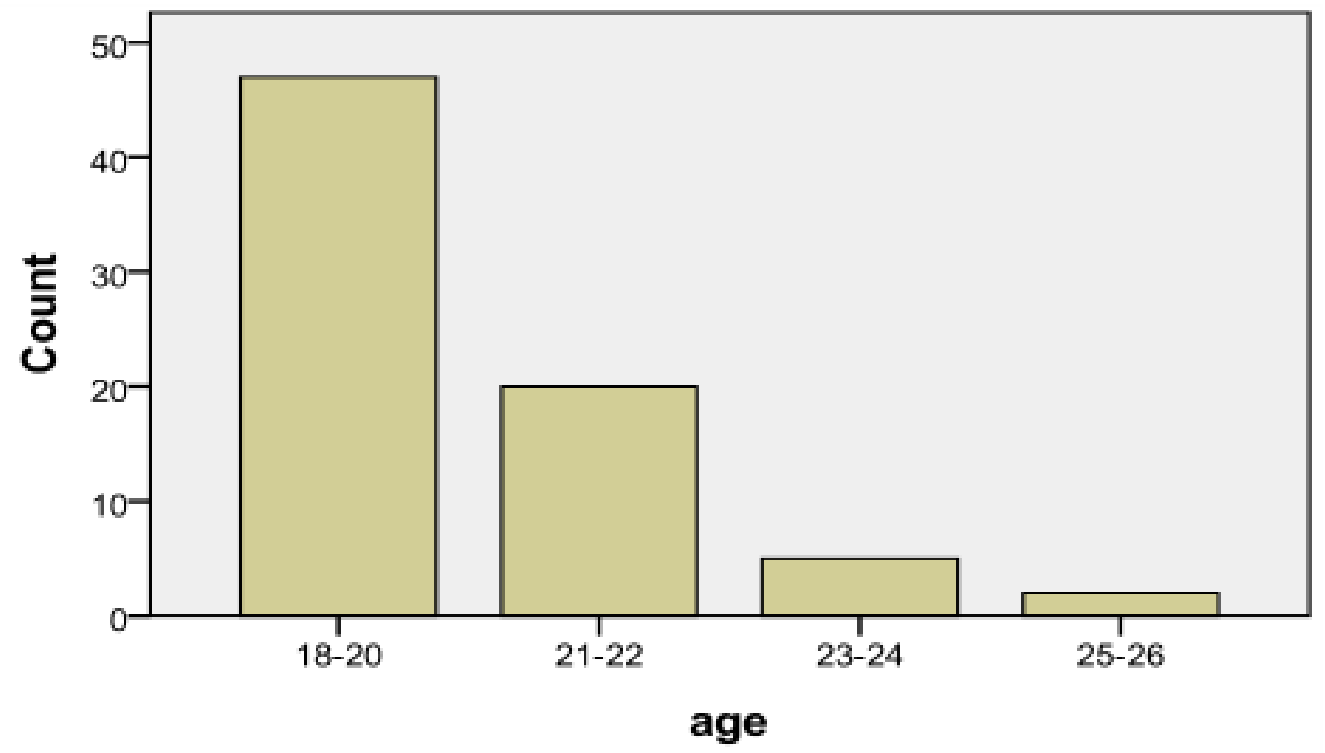


Table Distribution of sample and percentage on origin $(\mathrm{N}=74)$

Table No.4.1.3

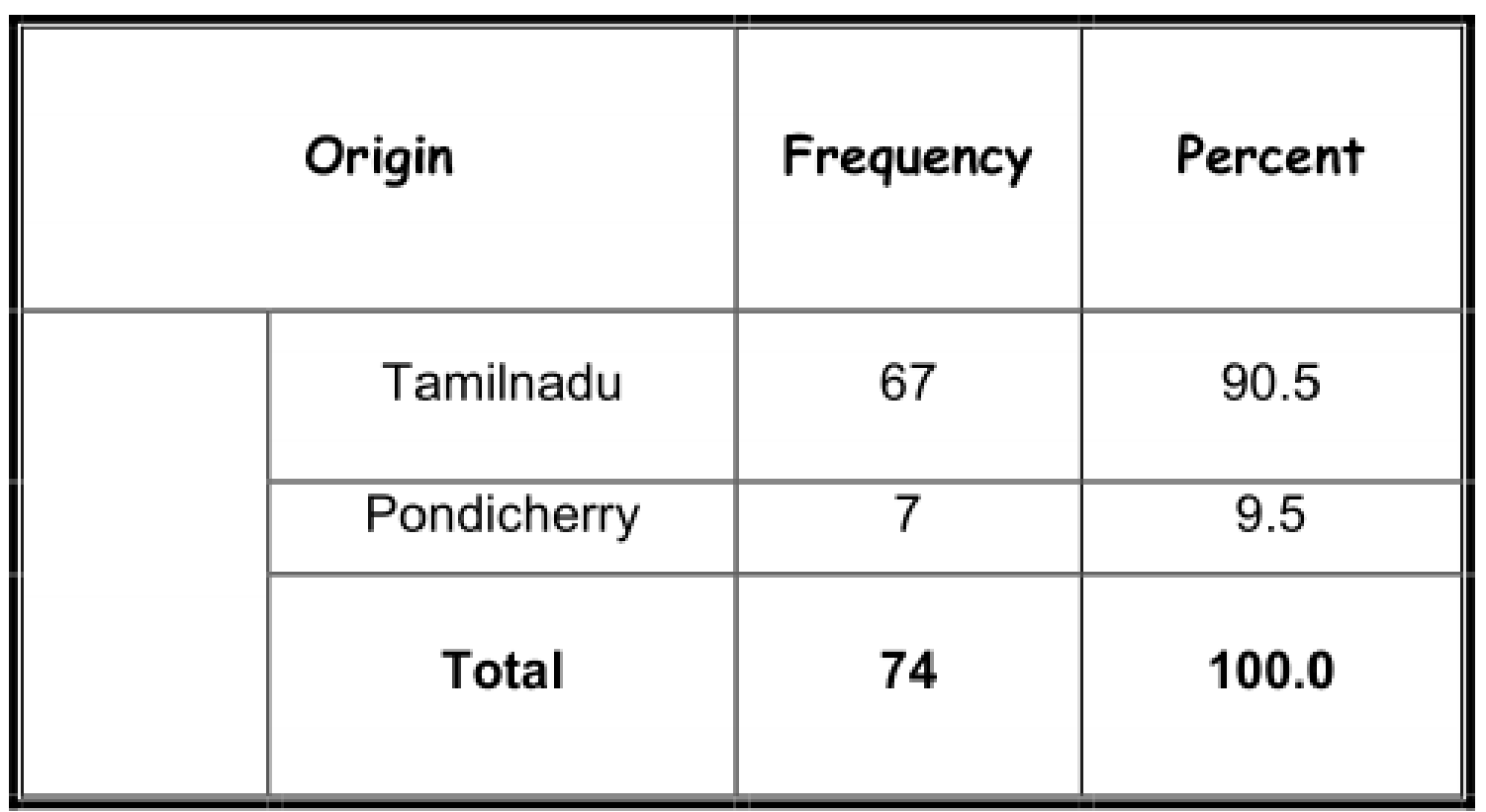

Figure Bar diagram showing the distribution of sub samples based on th origin of students $(\mathrm{N}=74)$

Graph No.4.1.3

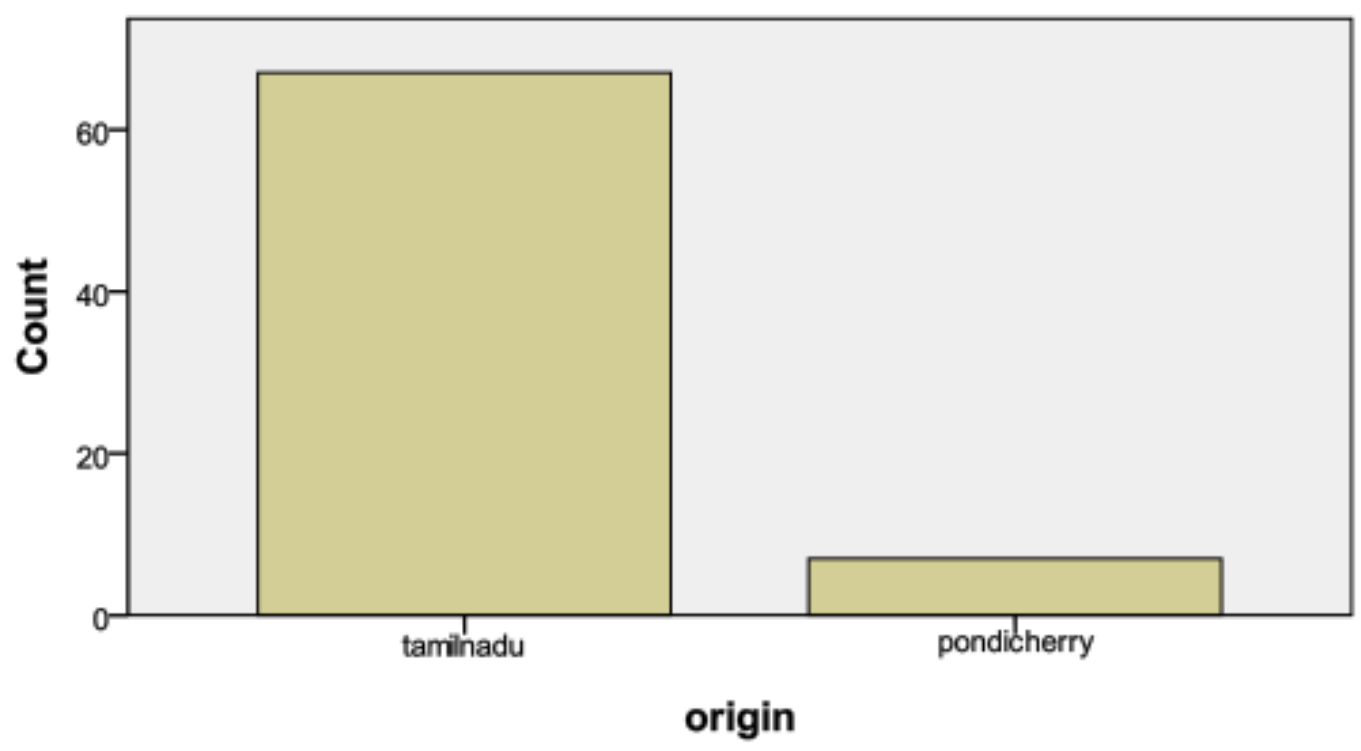

Table Distribution of sample and percentage on marks $(\mathrm{N}=74)$ 
Table Distribution of sample and percentage on marks $(N=74)$

Table No.4.1.4

\begin{tabular}{||l|l|r|r|}
\hline \hline \multicolumn{2}{|c|}{ Marks } & Frequency & \multicolumn{2}{|c|}{ Percent } \\
\hline \multirow{4}{*}{ Valid } & $500-700$ & 9 & 12.2 \\
\cline { 2 - 4 } & $701-900$ & 32 & 43.2 \\
\cline { 2 - 4 } & $901-1100$ & 20 & 27.0 \\
\cline { 2 - 4 } & $1101-1200$ & 5 & 6.8 \\
\cline { 2 - 5 } & Total & 66 & 89.2 \\
\hline Missing & System & 8 & 10.8 \\
\hline Total & & 74 & 100.0 \\
\hline
\end{tabular}

The above table represents the subject marks. The sample was divided into four groups according to marks. The table reveals that 9 subjects were under 500-700 of mark (12.2\%), 32 subjects were under $701-900$ of mark (43.2\%), 20 subjects were under $901-1100$ of mark (27\%), 5 subjects were under 1101 1200 of marks (6.8\%).

Figure Bar diagram showing the distribution of sub samples based on the marks of students $(\mathrm{N}=74)$ Graph No.4.1.4

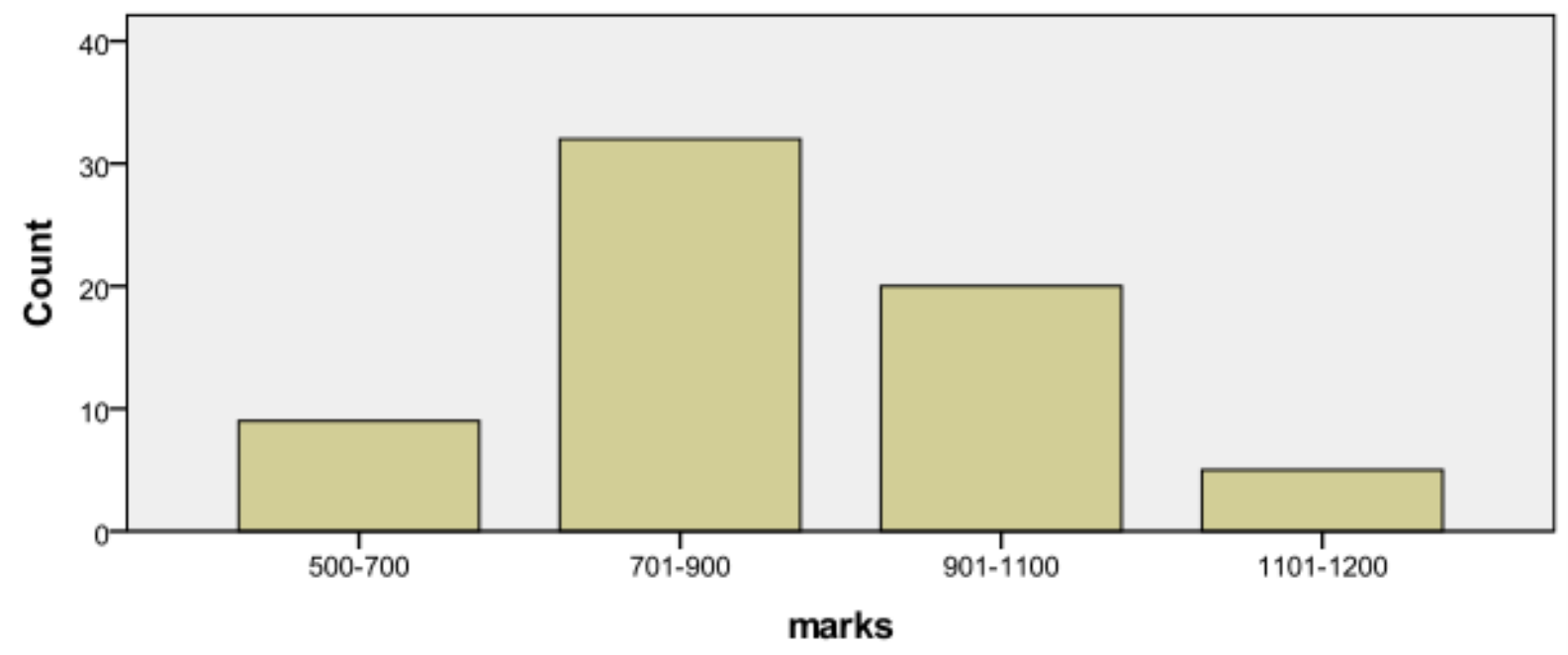


International Journal of Trend in Scientific Research and Development (IJTSRD) ISSN: 2456-6470

Table Distribution of sample and percentage on birth order $(N=74)$

Table No.4.1.5

\begin{tabular}{|c|c|c|c|}
\hline \multicolumn{2}{|c|}{ Birth order } & Frequency & Percent \\
\hline Valid & 1.00 & 29 & $\overline{39.2}$ \\
\hline & 2.00 & 26 & 35.1 \\
\hline & 3.00 & 11 & 14.9 \\
\hline & Total & 66 & 89.2 \\
\hline Missing & System & 8 & 10.8 \\
\hline Total & & 74 & 100.0 \\
\hline
\end{tabular}

Figure Bar diagram showing the distribution of sub samples based on the birth order of students $(\mathrm{N}=74)$ Graph No.4.1.5

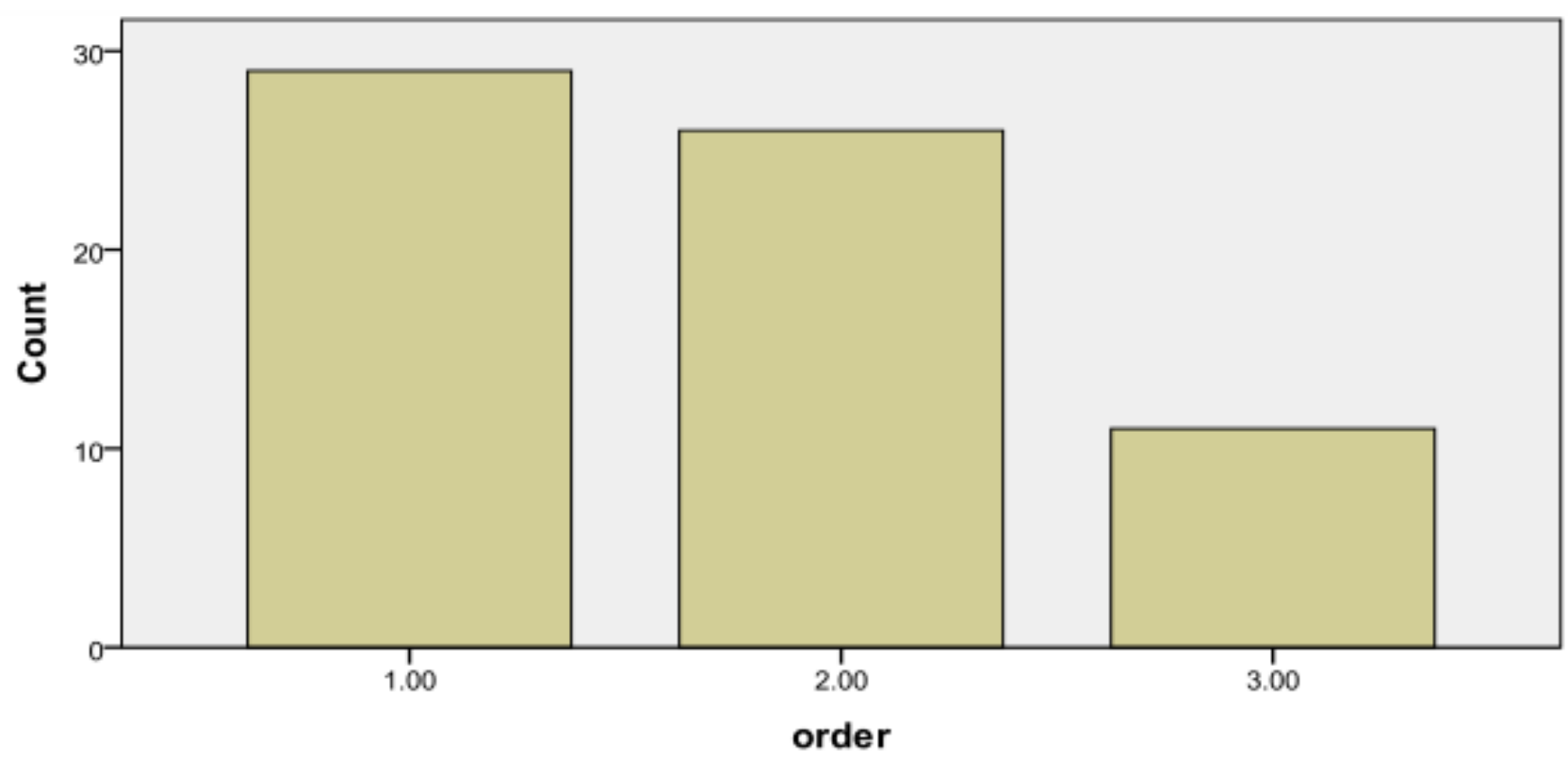


Table Distribution of sample and percentage on residence $(N=74)$

Table No.4.1.6

\begin{tabular}{|c|c|c|c|}
\hline & Residence & Frequency & Percent \\
\hline Valid & $\begin{array}{l}\text { with parents } \\
\text { hostel } \\
\text { Total }\end{array}$ & $\begin{array}{r}68 \\
6 \\
74\end{array}$ & $\begin{array}{r}91.9 \\
8.1 \\
100.0\end{array}$ \\
\hline
\end{tabular}

Figure Bar diagram showing the distribution of sub samples based on the residence of students $(\mathrm{N}=74)$ Graph No.4.1.6

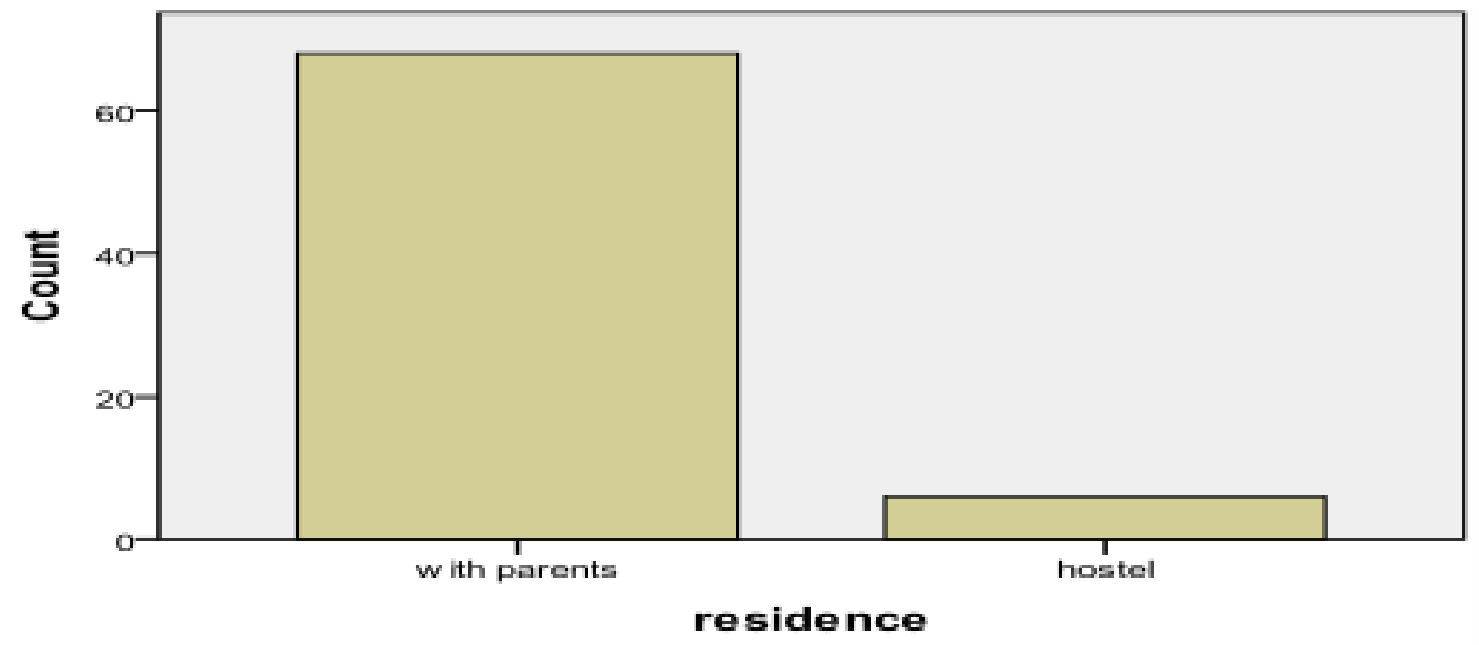

Table shows descriptive statistic for demographic data 
International Journal of Trend in Scientific Research and Development (IJTSRD) ISSN: 2456-6470

Table shows descriptive statistic for demographic data

\section{Table No.4.1.7}

\begin{tabular}{|c|c|c|c|c|c|}
\hline & $\mathrm{N}$ & $\begin{array}{c}\text { Minim } \\
\text { um }\end{array}$ & Maximum & Mean & Std. Deviation \\
\hline Age & 74 & 1.00 & 4.00 & 1.4865 & .74472 \\
\hline Sex & 74 & 1.00 & 1.00 & 1.0000 & .00000 \\
\hline Course & 74 & 1.00 & 4.00 & 2.3919 & .97668 \\
\hline Semester & 74 & 2.00 & 6.00 & 4.1351 & 1.51995 \\
\hline Marital & 74 & 2.00 & 2.00 & 2.0000 & .00000 \\
\hline Children & 47 & 1.00 & 5.00 & 2.5319 & .92903 \\
\hline Order & 66 & 1.00 & 3.00 & 1.7273 & .73475 \\
\hline Origin & 74 & 1.00 & 2.00 & 1.0946 & .29465 \\
\hline Marks & 66 & 1.00 & 4.00 & 2.3182 & .80688 \\
\hline Residence & 74 & 1.00 & 2.00 & 1.0811 & .27482 \\
\hline Valid N & 44 & & & & \\
\hline
\end{tabular}

The above Table shows Descriptive statistics on age, sex, course, semester, marital, children, order, origin, marks, Residence of the variables drinking motives and self esteem of the students 


\section{DRINKING MOTIVES}

Table Different drinking motives of the students $(\mathrm{N}=74)$

Table No.4.1.8

\begin{tabular}{|c|c|c|c|c|c|}
\hline & & $\mathrm{N}$ & & & \\
\hline & $\begin{array}{l}\text { Legally } \\
\text { valid }\end{array}$ & Missing & Mean & SD & $\begin{array}{l}\text { Cronbach } \\
\text { alpha }\end{array}$ \\
\hline Enhancement drink & 74 & 0 & 2.9405 & 1.28499 & .92 \\
\hline Coping drink & 74 & 0 & 2.9514 & 1.35679 & .90 \\
\hline Confirmatory drink & 74 & 0 & 2.9135 & 1.32130 & .90 \\
\hline Social drink & 74 & 0 & 3,0595 & 1.34541 & .92 \\
\hline
\end{tabular}

The above table indicates that mean, standard deviation, and crobach's alpha of the variable drinking motives. It indicates enhancement motive gets mean, median, standard deviation are 2.9405 , and 1.28499 respectively, coping motive gets mean, median, standard deviation are 2.9514, and 1.35679 respectively, confirmatory motives gets mean, median, standard deviation are 2.9135 , and 1.32130 respectively, social motive get mean, median, and standard deviation are 3.0595 , and 1.34541 respectively. The reliabilities for enhancement, coping, confirmatory, and social motive are $.92, .90,90$, and .92 respectively

\section{FINDINGS, CONCLUSION}

In this present study, data were collected from 74 students studying in three colleges in Chennai. Students from each college were chosen on purposive sampling from three colleges were included in this study. Among them 47 were 20 and below 20 and 27 were above 20 years. 25 students belong to engineering, 23 belong to nursing and 26 belong to arts and Sciences College. The Drinking Motives Questionnaire-Revised (DMQ-R; Cooper, 1994) was used to explore motives of drinking.
AND MAJOR FINDINGS

$>$ (18-20) age group( $\mathrm{n}=47)$ had more drinking problems compared to other age groups

43.2 percent students who drinks alcohol belongs to (701-900) marks category

$>39.2$ percent of samples who drinks belong to first order of birth

Majority of students 91.9 percent lived with parent during data collection

Social motive and coping motive were the top motives for the alcohol consumption followed by enhancement and confirmatory drink

$>$ None of the demographic variable had association with the drinking motives 


\section{IMPLICATIONS OF THE STUDY}

The outcome of any research depends upon the extent to which it has widened the existing frontiers of knowledge. It is also depends upon the degree of its social relevance and utility. The present investigation has shown that the drinking motives and demographic variable of students can be significantly related with each other.

\section{SUGGESTIONS FOR FURTHER RESEARCH}

1. Further research may be conducted with large representative samples of the undergraduate students.

2. Further, a comparative study may be conducted with male students and female students so as to find out a gender difference.

3. The study may be conducted with the students of other higher educational institutions so as to get the conformity of results.

4. Further research conducted with both samples of undergraduates students and post graduate students

5. Further studies may be conducted to explore the relationship between drinking motives and other psychological variables such as personality, motivation, decision making, and emotional intelligence.

\section{LIMITATIONS OF THE STUDY}

1. The present study was confined to students in the regular colleges of the Chennai city.

2. The study was confined only to male undergraduate students.

3. The samples were confined to only three colleges in Chennai with only two variables.

\section{CONCLUSION}

Our findings strongly support motivational approaches to understanding problem drinking among college students and are consistent with previous research in showing drinking motives to be a crucial determinant of college student drinking. Enhancement, coping and confirmatory motive seemed to be the most important factor in Chennai students drinking behavior.

These findings have several implications for public health research and interventions. There is need for a continued focus on individual Enhancement, coping and confirmatory motive reasons for drinking in adolescents and young adults in substance use prevention programs. Alcohol motives are likely to have been shaped by other indirect and distal forces such as availability and the media. For example, television scenes that glamorize its use or incorporate strong symbolic meaning, such as rebellion against prejudice, while featuring alcohol may cause young people to initiate and continue drinking. Research that Offers a better understanding of psycho-social and environmental factors associated with alcohol use behavior among the younger population in Chennai is urgently needed.

\section{Source of funding - NIL}

This dissertation submitted to PRIST UNIVERSITY in partial fulfillment of the requirement for the award of the degree of Mphil sociology.

\section{REFERENCES}

1. Abbey, A., Smith, M. J., \& Scott, R. O. (1993). The relationship between reasons for drinking alcohol and alcohol consumption: An interactional approach. Addictive Behaviors, 18(6), 659-670.

2. Alvarez, F. J., \& Del Rio, M. C. (1994). Gender differences in patterns of alcohol consumption in Spain. Alcoholism, Clinical and Experimental Research, 18(6), 1342-1347.

3. Armeli, S., Carney, M. A., Tennen, H., Affleck, G., \& O'Neil, T. P. (2000). Stress and alcohol use: A daily process examination of the stressorvulnerability model. Journal of Personality and Social Psychology, 78, 979 - 994.

4. Blascovich, J., \& Tomaka, J. (1991). Measures of self-esteem. In J. P. Robinson, P. R. Shaver, \& L. S. Wrightsman (Eds.) Measures of personality and social psychological attitudes, Volume I. San Diego, CA: Academic Press.

5. Boys, A., Marsden, J., \& Strang, J. (2001). Understanding reasons for drug use amongst young people: A functional perspective. Health Education Research, 16(4), 457-469.

6. Bradley, J. R., Carman, R. S., \& Petree, A. (1991). Expectations, alienation, and drinking motives among college men and women. Journal of Alcohol and Drug Education, 21(1), 27-33-44

7. Campbell, J. D., \& Lavalee, L. F. (1993). Who am I? The role of self-concept confusion in understanding the behavior of people with low self-esteem. In R.F. Baumeister (Ed.), Selfesteem. The puzzle of low self-regard (pp 3-20 
8. Carpenter, K. M., \& Hasin, D. S. (1998b). Reasons for drinking alcohol: Relationships with DSM-IV alcohol diagnoses and alcohol consumption in a Community Sample. Psychology of Addictive Behaviors, 12(3), 168-18.

9. Chastain, G. (2006). Alcohol, neurotransmitter systems, and behavior. The Journal of General Psychology, 133(4), 329-335.

10. Cooper, M. L. (1994). Motivations for alcohol use among adolescents: Development and validation of a four-factor-model. Psychological Assessment, 6(2), 117-128.

11. Cooper, M. L., Frone, M. R., Russell, M., \& Mudar, P. (1995). Drinking to regulate positive and negative emotions: A motivational model of alcohol use. Journal of Personality and Social Psychology, 69(5), 990-1005.

12. Cooper, M. L., Russell, M., Skinner, J. B., \& Windle, M. (1992). Deyelopment and validation of a three-dimensional measure of drinking motives. Psychological Assessment, 4, 123-132.

13. Cooper, M. L. (1994). Motivations for alcohol use among adolescents: Development and validation of a four-factor-model. Psychological Assessment, 6(2), 117-128.

14. Coopersmith, S. A. $(1967,1981)$. The antecedents of self-esteem. San Francisco Freeman.Cox, W. M., \& Klinger, E. (1988). A motivational model of alcohol use. Journal of Abnormal Psychology, 97(2), 168-180.

15. Cronin, C. (1997). Reasons for drinking versus outcome expectancies in the prediction of college student drinking. Substance Use and Misuse, 32(10), 1287-1311.

16. De Micheli, D., \& Formigoni, M. L. (2002). Are reasons for the first use of drugs and family circumstances predictors of future use patterns? Addictive Behaviors, 27(1), 87-100. 